Draft version April 6, 2021

Typeset using $\mathrm{LAT}_{\mathrm{E}} \mathrm{X}$ twocolumn style in AASTeX63

\title{
Narrowband Spikes Observed during the 2013 November 7 Flare
}

\author{
Marian KarlickÝ, ${ }^{1}$ Jan BenáČeK, ${ }^{2}$ And JÁn RybÁK ${ }^{3}$ \\ ${ }^{1}$ Astronomical Institute of the Academy of Sciences of the Czech Republic, Fričova 298, CZ-25165 Ondřejov, Czech Republic \\ ${ }^{2}$ Center for Astronomy and Astrophysics, Technical University of Berlin, 10623 Berlin, Germany \\ ${ }^{3}$ Astronomical Institute, Slovak Academy of Sciences, SK-05960 Tatranská Lomnica, Slovakia
}

\begin{abstract}
Narrowband spikes are observed in solar flares for several decades. However, their exact origin is still discussed. To contribute to understanding of these spikes, we analyze the narrowband spikes observed in the 800-2000 MHz range during the impulsive phase of the November 7, 2013 flare. In the radio spectrum, the spikes started with typical broadband clouds of spikes, and then their distribution in frequencies changed into unique, very narrow bands having non-integer frequency ratios. We successfully fitted frequencies of these narrow spike bands by those, calculating dispersion branches and growth rates of the Bernstein modes. For comparison, we also analyzed the model, where the narrow bands of spikes are generated at the upper-hybrid frequencies. Using both models, we estimated the plasma density and magnetic field in spike sources. Then the models are discussed, and arguments in favor of the model with the Bernstein modes are presented. Analyzing frequency profiles of this spike event by the Fourier method, we found the power-law spectra with the power-law indices varying in the -0.8 -2.75 interval. Because at some times this power-law index was close to the Kolmogorov spectral index $(-5 / 3)$, we propose that the spikes are generated through the Bernstein modes in turbulent plasma reconnection outflows or directly in the turbulent magnetic reconnection of solar flares.
\end{abstract}

Keywords: Space plasmas - Solar radio flares - Radio bursts

\section{INTRODUCTION}

Radio bursts are an integral part of solar flares. Their types and basic characteristics are well described, e.g., in books by McLean \& Labrum (1985); Krueger (1979) and papers by Isliker \& Benz (1994) and Jiřička et al. (2001). Among these bursts the narrowband dm-spikes belong to those of the most interesting, as they seem to be connected with the primary flare energy-release processes (Droege 1977; Karlický 1984; Fu et al. 1985; Staehli \& Magun 1986; Guedel 1990; Krucker \& Benz 1994; Aschwanden et al. 1998; Zlobec \& Karlický 1998; Bouratzis et al. 2016). On the radio spectrum, spikes occur in clouds of many short duration narrowband bursts with a typical relative bandwidth of about 1-3

karlicky@asu.cas.cz

benacek@tu-berlin.de

rybak@astro.sk
$\%$, duration less than $100 \mathrm{~ms}$ and the brightness temperature up to $10^{15} \mathrm{~K}$ (Benz 1986). In some cases, the narrowband spikes are observed near the starting frequency of type III bursts and in a good correlation with the hard X-ray emission (Dabrowski \& Benz 2009).

Several models of the narrowband spikes were proposed. For example, in papers by Kuijpers et al. (1981); Tajima et al. (1990); Wentzel (1991) the runaway electrons, accelerated in a strong DC electric field, are considered as the primary source of the spikes. In other models, the electron-cyclotron maser (ECM) mechanism in connection with the loss-cone distribution of superthermal electrons was suggested. In papers by Melrose \& Dulk (1982), Vlahos \& Sharma (1985), Winglee et al. (1988), Aschwanden (1990), Fleishman \& Mel'nikov (1998), Fleishman et al. (2003) and Melrose (2017) assuming the ratio $Y=\omega_{\mathrm{pe}} / \omega_{\mathrm{ce}}<1$, where $\omega_{\mathrm{pe}}$ and $\omega_{\mathrm{ce}}$ are the electron plasma and electron cyclotron frequencies, the electromagnetic waves (spikes) are generated directly by this ECM mechanism. In first versions of this mechanism the emis- 
sion occurs near the cyclotron frequency and its harmonics. However, Krucker \& Benz (1994) presented observations, where the narrowband spikes were clustered in bands with the non-integer ratio in the interval 1.06 - 1.54. Therefore, Willes \& Robinson (1996) proposed the model, where the spike frequencies correspond to the Bernstein modes (BM model). Similarly, Stepanov et al. (1999) and Bárta \& Karlický (2001) presented the model of spikes with the upper-hybrid waves (UHW model). In these models the ratio $Y$ is greater than unity. We note that in some conditions also the direct ECM mechanism may generate non-integer harmonic ratios (Fleishman \& Mel'nikov 1998). Recently, using the particle-in-cell model, the plasma emission induced by the ECM instability in solar plasmas with the ratio $Y=10$ have been studied by $\mathrm{Ni}$ et al. (2020). They found that the fundamental emission is caused by coalescence of almost counterpropagating Z-modes and whistlers, while the harmonic emission arises from coalescence of an almost counterpropagating UH waves.

For understanding of these spikes also the bandwidth and duration of individual spikes were studied in details. For example, in the paper by Rozhansky et al. (2008) the characteristic spike half-maximum duration $\tau$ in dependence of the frequency $f$ was derived as $\tau \sim f^{-1.29}$. On the other hand, the power-law distributions of spike bandwidths in dependence on frequency were found by Nita et al. (2014).

In papers by Karlický et al. (1996, 2000), the frequency bandwidths of spikes were studied by the Fourier method. As a result the power-law spectra with powerlaw indices in the range of $-0.80--2.85$ were found. Similar results were presented by Messmer \& Benz (2000). Because in several events the power-law indices were close to $-5 / 3$, it was suggested that the narrowband dm-spikes are generated by superthermal electrons in the magnetohydrodynamic (MHD) turbulence in the magnetic reconnection outflows (Karlický et al. 1996; Zlobec \& Karlický 1998).

Magnetic reconnection converts magnetic energy to other forms of energy, driving thus solar flares and accelerating particles (Priest \& Forbes 2000). Its 2-D numerical models have shown that a current layer during the reconnection becomes fragmented to smaller and smaller magnetic islands (plasmoids) (Loureiro et al. 2007; Bhattacharjee et al. 2009; Bárta et al. 2011a,b; Karlický \& Bárta 2011). On the other hand, 3-D numerical simulations (Drake et al. 2019) show evolution of turbulence in agreement with the turbulent reconnection theory (Lazarian \& Vishniac 1999; Lazarian et al. 2020). Thus, the turbulence can take place directly in the reconnection layer as well as in the plasma reconnection outflows. In turbulent reconnection the electrons are effectively accelerated as described by Dahlin et al. (2014), Guo et al. (2015), Zhou et al. (2016) and Guo et al. (2020).

In the present paper, we analyze the narrowband spikes observed during the impulsive phase of the November 7, 2013 flare. In the radio spectrum, these spikes firstly appear in clouds of spikes as in typical spike events, but then the frequency distribution of these spikes changed to unique, very narrow bands with the non-integer frequency ratios. Just these very narrow bands of spikes with the non-integer frequency ratios enable us to verify some their models. Calculating dispersion branches and growth rates from analytical equations, we show that these spikes can be explained by the model with the Bernstein modes proposed by Willes \& Robinson (1996). Using this model, we determine the plasma density and magnetic field strength in a source of the spikes. For comparison, we also computed the growth rates of the upper-hybrid waves in the models proposed by Stepanov et al. (1999) and Bárta \& Karlický (2001). Comparing the both models, we conclude that the model with the Bernstein modes is more probable. We note that the transition from typical broadband clouds of spikes to very narrow spike bands, observed in this spike event, shows that the results obtained in the analysis of these narrow bands have also a general significance at least for some group of spike events. In this paper, we also analyze frequency profiles of these spikes by the Fourier method. Based on the computed power-law spectra, we suggest that the spikes are generated in turbulent plasma reconnection outflows or directly in turbulent magnetic reconnection of solar flares.

\section{OBSERVATIONS AND THEIR ANALYSIS}

During 1992-2020 years the Ondřejov radiospectrographs (Jiřička et al. 1993; Jiřička \& Karlický 2008), operating in the $800-2000 \mathrm{MHz}$ range, registered 53 clouds of the narrowband spikes. Two examples of the narrowband spikes observed in the impulsive phase of the 14 September 1992 and 10 June 2003 flares are shown in Figure 1. As can be seen here the narrowband spikes are typically clustered in broad frequency bands; in these cases at least in two bands. In the 10 June 2003 radio spectrum the narrowband spikes are localized close to the starting frequency of type III bursts, see Figure $1 \mathrm{~b}$ at frequencies below $1200 \mathrm{MHz}$. It indicates that the narrowband spikes are generated close to the region where electrons are accelerated.

Among these 53 cases of the observed narrowband spikes, we found a unique example of the narrow- 
band spikes observed in November 7, 2013 (Figure 2). The time and frequency resolution of the radio spectrum in this case is $0.01 \mathrm{~s}$ and $4.7 \mathrm{MHz}$, respectively (Jiřička \& Karlický 2008). These spikes started at 12:26:09 UT with the broad bands of spikes. Around 12:26:14 UT these spikes are so densely distributed that nearly form a continuum. Then the density of spikes in the radio spectrum decreases and varies up to 12:26:39 UT. Whole this interval of spikes from 12:26:09 till 12:26:39 UT resembles to typical cases of the narrowband spikes in this frequency range. After 12:26:39 UT four bands of spikes appeared and they were very narrow. The spike event ends at about 12:27:20 UT. The part of the spike event with very narrow bands of spikes is unique as well as the transition from broadband bands of spikes to narrow ones. As will be seen in the following, the part of the spike event with four very narrow bands of spikes enables comparison with theoretical models of the narrowband spikes. On the other hand the transition from broad bands of spikes to very narrow bands shows that the results obtained for spikes in the narrow bands are also valid for spikes at the beginning part of the spike event (at 12:26:09 till 12:26:39 UT) that resembles to typical spike events.

These narrowband spikes presented in Figure 2a were observed in the impulsive phase of the November 7, 2013 flare that occurred in the NOAA AR 1890 in $\mathrm{H}_{\alpha}$ with the start at 12:17 UT, maximum at 12:28 UT, and end at 12:44 UT, and in GOES soft X-ray emission with the start at 12:22 UT, maximum at 12:29 UT, and end at 12:34 UT. At these times the Atmospheric Imaging Assembly (AIA) (Lemen et al. 2012) onboard the Solar Dynamic Observatory (SDO) (Pesnell et al. 2012) shows a compact flare with many interacting multi-thermal loops. There is a gap in RHESSI hard X-ray observations.

Details of the very narrow bands of spikes with the maximal time resolution $(0.01 \mathrm{~s})$ and in the time interval of one second starting at 12:26:55 UT are shown in Figure $2 \mathrm{~b}$ and $2 \mathrm{c}$. These spectra are without any smoothing and thus showing bins with the duration $0.01 \mathrm{~s}$ and frequency width $4.7 \mathrm{MHz}$. The characteristic half-maximum duration of spikes in the frequency range under study is about $0.01 \mathrm{~s}$ (Mészárosová et al. 2003; Rozhansky et al. 2008). On the other hand, the mean bandwidth of individual spikes was reported by Nita et al. (2014) as 7.5 MHz. Considering these values and time and frequency resolution of the spectra, it means that bright bins roughly correspond to individual spikes. It also means that these spikes are typical spikes. However, in this event they are clustered in unique, very narrow bands, where individual spikes are overlapping in some locations. The frequency of these narrow bands at 12:26:55 UT is about 1003, 1276, 1572 and $1877 \mathrm{MHz}$. It gives the ratio between frequencies of the neighboring bands as $1877 \mathrm{MHz} / 1572 \mathrm{MHz}=1.19$, $1572 \mathrm{MHz} / 1276 \mathrm{MHz}=1.23$, and $1275 \mathrm{MHz} / 1000 \mathrm{MHz}$ $=1.275$. For comparison, the frequency ratio between neighboring spike bands reported by Krucker \& Benz (1994) is in the 1.06-1.54 range. Thus, our values of this ratio are within this range. Further interesting aspect of these narrow bands can be seen in Figure 2b. Here, in the time interval 12:26:55.2 - 12:26:55.4 UT there is the band frequency variation (see the arrow), form of which is synchronized with the similar forms in all four bands. No further narrow bands at this time and at frequencies below $800 \mathrm{MHz}$ (Callisto BLEN 200-900 MHz spectrum $^{1}$ ) and above $2000 \mathrm{MHz}$ (Ondřejov 2000-5000 $\mathrm{MHz}$ spectrum) were found.

Figure 3a shows the radio flux evolution in the time interval $1 \mathrm{~s}$ starting at 12:26:55 UT at four frequencies $(1003,1276,1572$ and $1877 \mathrm{MHz})$ that approximately cut the bands of spikes presented in Figure 2b. In all these radio flux records, there are variations on the shortest time scale $0.02 \mathrm{~s}$ (twice the temporal resolution). To know the relation between spikes in these bands with the shortest time resolution $(0.01 \mathrm{~s})$, we calculated cross-correlations between the radio flux profiles at these bands. The maximum cross-correlations are shown in Figure 3b: 0.38 between the radio fluxes at frequencies $1276 \mathrm{MHz}$ and $1003 \mathrm{MHz}$ (blue line), 0.35 at frequencies $1572 \mathrm{MHz}$ and $1276 \mathrm{MHz}$ (red line), and 0.28 at frequencies $1877 \mathrm{MHz}$ and $1572 \mathrm{MHz}$ (black line). In two cross-correlations the time lag was zero (black and blue line), and in the third case (red line) the time lag was $0.01 \mathrm{~s}$ (profile at $1572 \mathrm{MHz}$ was delayed $0.01 \mathrm{~s}$ after that on $1276 \mathrm{MHz}$ ). The cross-correlations between channels from not neighboring spike bands were lower. The maximum cross-correlations are low, caused by the low value of the signal/noise ratio at this $0.01 \mathrm{~s}$ time resolution and overlapping of spikes, but with the peaks clearly visible. Moreover, as mentioned above there are variations of band frequencies that are synchronized in all four bands.

We also made 10 profiles of the radio flux vs. frequency for the spikes observed during 12:26:55.00 12:26:55.09 UT (Figure 4a). The time difference between neighboring profiles is $0.01 \mathrm{~s}$. Auto-correlations of these profiles, showing the frequency lag of about 300 $\mathrm{MHz}$, are shown in Figure 4b.

\footnotetext{
${ }^{1}$ http://www.e-callisto.org/
} 


\section{MODELS}

Owing to a very narrow bandwidth of the four bands of spikes and non-integer ratio of their neighboring frequencies, the 7 November 2013 event is a good example for model verification. Because of this non-integer ratio and because in the solar corona the mean ratio $\omega_{\text {pe }} / \omega_{\text {ce }}$ is of the order of unity or larger (see the models of the plasma density and magnetic field in the corona in the book by Aschwanden (2004)), in the following, we analyze the models that are relevant to these conditions. (However, it is not possible to exclude that in some localized regions in the solar corona this ratio is lower than unity.)

First, we consider the model by Willes \& Robinson (1996), where the observed band frequencies of spikes are the Bernstein mode frequencies (BM model). We calculate dispersion branches of the Bernstein modes and their corresponding growth rates in a similar way as in the paper by Benáček \& Karlický (2019). We assume a plasma that consists of a Maxwellian cold and dense background plasma and rare hot superthermal electrons having the loss-cone DGH distribution (Dory et al. 1965). Such a plasma is unstable as shown in Appendix A, where the analytical relations for computations of the electrostatic dispersion branches together with their growth rates are described in detail. Using these relations, we tried to fit the observed spike band frequencies shown in Figure 2b (1003, 1276, 1572 and $1877 \mathrm{MHz}$ ) in such a way that the dispersion branches intersect the positive growth rate regions just at the observed frequencies. Plasma parameters for this fitting procedure were taken to be appropriate to solar flare conditions. A good agreement between the observed and model frequencies was found for the following parameters: $\omega_{\mathrm{pe}} / \omega_{\mathrm{ce}}=2.7, v_{\mathrm{tb}} / c=0.02(2.38 \mathrm{MK})$, $v_{\mathrm{t}} / c=0.25$, and $n_{\mathrm{e}} / n_{\mathrm{h}}=10$, where $v_{\mathrm{tb}}$ is the thermal velocity of the Maxwellian background plasma, $v_{\mathrm{t}}$ is the characteristic velocity of the superthermal electrons, $c$ is the speed of light, and $n_{\mathrm{e}}$ and $n_{\mathrm{h}}$ are the background cold plasma and hot plasma densities.

The spikes in BM model can be generated on BM frequencies by the process $\mathrm{BM} \pm \mathrm{S} \rightarrow \mathrm{T}$, where $\mathrm{BM}$ is the Bernstein mode, $\mathrm{S}$ is the low-frequency wave and $\mathrm{T}$ is the transversal electromagnetic (radio) wave, or on the double BM frequencies by the process BM $+\mathrm{BM}$ $\rightarrow$ T. Willes \& Robinson (1996) preferred the emission on the double BM frequencies saying that this process does not require additional low-frequency waves. However, as shown by $\mathrm{Ni}$ et al. (2020) the low-frequency waves (whistlers and ion-acoustic) are present in such processes. Therefore in the following estimations of the plasma density and magnetic field, we consider both the possible processes.

The result of the above described fitting procedure with the assumption that the radio emission frequencies of the spike bands correspond to BM frequencies is shown in Figure 5, i.e. the frequencies in $\mathrm{MHz}$ are given for $\omega_{\mathrm{pe}}=2 \pi f_{\mathrm{pe}}, f_{\mathrm{pe}}=855 \mathrm{MHz}$. (We note that for the emission on the double BM frequencies the values of $f$ in $\mathrm{MHz}$ should be divided by 2 and $f_{\mathrm{pe}}=427.5$ $\mathrm{MHz}$.) Positive growth rates in this figure are expressed by the blue-red regions that are approximately at harmonics of the cyclotron frequency. When the dispersion branch (green line) intersects a positive growth rate region, the Bernstein (electrostatic) modes are generated. In our case it is for the Bernstein modes with the gyroharmonic number $s=3,4,5$, and 6 , corresponding to the spike band frequencies $1003 \mathrm{MHz}, 1276 \mathrm{MHz}, 1572$ $\mathrm{MHz}$ and $1877 \mathrm{MHz}$, respectively. While the fit of three bands $(1276,1572$ and $1877 \mathrm{MHz})$ is nearly exact, there is some deviation between the observed band frequency at about $1003 \mathrm{MHz}$ and that found by fitting $950 \mathrm{MHz}$. But, note that this branch is close to the frequency gap in observations and the parameters in computations can also deviate from real ones.

Now taking the spike band frequencies as BM frequencies and using the relations $f_{\mathrm{pe}}[\mathrm{MHz}]=9 \times$ $10^{-3} \sqrt{n_{e}\left[\mathrm{~cm}^{-3}\right]}$ and $f_{\mathrm{ce}}[\mathrm{MHz}]=2.8 \times B[\mathrm{G}]$, where $f_{\text {ce }}=\omega_{\text {ce }} / 2 \pi$ (Karlický \& Yasnov 2018) we estimated the mean plasma density and magnetic field strength in the spike source in the November 7, 2013 event as $n_{\mathrm{e}}=9$ $\times 10^{9} \mathrm{~cm}^{-3}$ and $B=113 \mathrm{G}$, respectively. On the other hand, for the radio emission on the double BM frequencies the values of the plasma density and magnetic field strength are 4 and 2 times lower, respectively.

Now for comparison, let us consider the model by Stepanov et al. (1999) and Bárta \& Karlický (2001). In this model the observed band frequencies of spikes are frequencies of UHW. Using the equations presented in Appendix A and varying the ratio $\omega_{\mathrm{UH}} / \omega_{\mathrm{ce}}$, we computed the growth rates of the upper-hybrid waves in the interval of the gyro-harmonic number $s=3-6$, see Figure 6. Other parameters were same as in the BM model. Each growth rate $\Gamma$ in this figure corresponds to the maximal growth rate on the upper-hybrid wave branch. The maximal growth rates are found for the ratios $\omega_{\mathrm{UH}} / \omega_{\mathrm{ce}}=2.84,3.80,4.75$, and 5.71. Although the parameters and approximations in the present computations of the growth rates differ from those used by Stepanov et al. (1999), the results are similar.

Contrary to the BM model proposed by Willes \& Robinson (1996), where all bands of spikes 
are generated in one source, in the UHW model, each spike band is generated in different regions with different ratios of the plasma density and the magnetic field. This model is similar to that proposed for zebra patterns (Zlotnik 2013; Karlický \& Yasnov 2018). Thus, generally spike bands could be used for determining the plasma density and magnetic field strength in spike band sources. But, a problem is how to determine the gyro-harmonic numbers of the spike bands in this case. If only as an example we assume that the frequencies of observed spike bands $f_{\mathrm{UH}}=1003,1276,1572$, and $1877 \mathrm{MHz}$ are the upper-hybrid frequencies for $s=3,4,5$ and 6 , then the plasma density and magnetic field strength in four sources corresponding to four bands in the 7 November 2013 event can be estimated as $n_{\mathrm{e}}=1.1 \times 10^{10}, 1.9 \times 10^{10}, 2.9 \times 10^{10}$, and $4.2 \times 10^{10} \mathrm{~cm}^{-3}$ and $B=119,114,112$, and $111 \mathrm{G}$, respectively. If on the other hand, in agreement with zebra pattern observations, we assume the inverse sequence of $s=6$, $5,4,3$ for these spike bands then the plasma density and magnetic field strength in these four sources are $n_{\mathrm{e}}=1.2 \times 10^{10}, 1.9 \times 10^{10}, 2.9 \times 10^{10}, 3.9 \times 10^{10} \mathrm{~cm}^{-3}$ and $B=60,91,140,223 \mathrm{G}$, respectively. Here, we used the relations $n_{\mathrm{e}}\left[\mathrm{cm}^{-3}\right]=f_{\mathrm{UH}}^{2}\left(1-1 / s^{2}\right) / 8.1 \times 10^{-5}$ and $B[\mathrm{G}]=f_{\mathrm{UH}} /(2.8 s)$, where $f_{\mathrm{UH}}$ is in $\mathrm{MHz}$ (Karlický \& Yasnov 2020). As follows from these estimations and relations the estimated plasma density is only partly dependent on the assumed gyro-harmonic number $s$. However, the magnetic field strongly depends on $s$, and for higher $s$ the magnetic field decreases. On the other hand, for the radio emission on the double UHW frequencies the values of the plasma density and magnetic field strength are 4 and 2 times lower, respectively. Comparing the estimated plasma density and magnetic field in the BM and UHW models, we can see that the plasma density and magnetic in the BM model is roughly the same as in the band on $1003 \mathrm{MHz}$ with $s$ $=3$ in UHW models, other values of the plasma density and magnetic field in the UHW model differ. But note that in BM model there is one source and in the UHW model four different sources. Moreover, we do not know the gyro-harmonic numbers of bands of spikes in the UHW model.

\section{DISCUSSIONS}

A question arises, which model better agrees with observed spikes: the BM model or the UHW model? The most important argument in favor of the BM model is that we succeeded (almost perfectly) to fit BM frequencies with the observed band structure. Moreover, the frequency difference among three bands was close to 300 MHz. It also speaks in favor of the BM model, because it is not very probable that the UHW model with three sources at different locations give such a result.

There is further argument in favor of the BM model. Namely, we found zero or $0.01 \mathrm{~s}$ time lag in the crosscorrelations between two neighboring spike bands (Figure $3 \mathrm{~b}$ ). Moreover, we found the band frequency variations synchronized in all four bands, see the arrow in Figure 2b. When we take now the UHW model, where band sources are at different locations, and taking the frequencies of spike bands and using the density model of the solar atmosphere by Aschwanden (2002), the distance between sources of neighboring spike bands can be estimated as about $2000 \mathrm{~km}$. When we assume the Alfvén waves for synchronization of the spikes in the bands, that are at the distance $2000 \mathrm{~km}$, within $0.01 \mathrm{~s}$ then the requested Alfvén speed is $200000 \mathrm{~km} \mathrm{~s}^{-1}$, which is unrealistic.

Now, let us look if some answers can be also found in an analysis of the bandwidth of individual spikes or bandwidth of spike bands. Namely, considering the BM model proposed by Willes \& Robinson (1996), we expect that increasing the gyro-harmonic number of Bernstein modes the bandwidth of individual spike as well as the bandwidth of spike bands increases roughly as an increase of the gyro-harmonic numbers, i.e., when we multiply the lowest and highest frequency of some individual spike or band of spikes by the gyro-harmonic number s, then also the difference between the lowest and highest frequencies (bandwidth) increases as multiplied by s. We cannot verify this effect on the bandwidth of individual spikes here because the frequency resolution of our observations is only $4.7 \mathrm{MHz}$ and the typical bandwidth of spikes in this range is $\sim 7.5 \mathrm{MHz}$ (Nita et al. 2014). However, Feng (2019) presented chains of spikes, where the bandwidth of the spikes on higher frequencies was about 2.1 times larger than that on lower frequencies. But, note that such an increase of the bandwidth of individual spikes in dependence on frequency can be also explained in models with direct ECM emission (Fleishman 2004).

Nevertheless, we can compare the bandwidth of the bands of spikes. Therefore in Figure 7, we show two contours of bands of narrowband spikes observed at about 12:26:15 UT. At this time two bands were recognized in the $1370-1620 \mathrm{MHz}$ and $1170-1370 \mathrm{MHz}$ range (red contour and red dashed horizontal lines) and 1370 - $1700 \mathrm{MHz}$ and 1370 - $1100 \mathrm{MHz}$ (blue contour and red dashed horizontal lines). Thus, the ratio of bandwidths of these two bands is $1.25(250 \mathrm{MHz} / 200 \mathrm{MHz})$ and $1.22(330 \mathrm{MHz} / 270 \mathrm{MHz})$ for red and blue bands, respectively. Because the ratio 1.25 (red band contours) corresponds to the ratio of gyro-harmonic numbers $s=5$ 
and $s=4(5 / 4=1.25)$ which were found for the very narrow bands that followed these bands of spikes (Figure 2a,b and 5), this result supports the BM model. However, this result needs to be taken with a caution because the bandwidth of spike bands can be influenced by an overlapping of these bands and also by variations of the spike intensities. We tried to check this result also in other spike events, but only a few of them were without these overlapping and thus appropriate for such an analysis. As a result, we found one example with the similar ratio as that presented here, and one opposite case, where the spike band on higher frequency was narrower than that on lower frequency, i.e., the ratio of these bands was less than 1. Summarizing this part of discussions, we think that BM model very well explains spikes in the November 7, 2013 event.

The present spikes were observed in the $800-2000 \mathrm{MHz}$ range during the impulsive flare phase. Moreover, in some cases the spikes appear close to the starting frequency of type III bursts (see Figure 1b). It indicates that the narrowband spikes in the $800-2000 \mathrm{MHz}$ range are closely connected with the primary flare energyrelease process, i.e., with the flare magnetic reconnection.

In papers by Karlický et al. $(1996,2000,2018)$ it was proposed that the spikes are generated in turbulence in the plasma reconnection outflows. This suggestion was based on the Fourier analysis of the spike frequency profiles. Therefore, let us make a similar analysis in the present case. Considering that spikes are generated on Bernstein modes then the spikes within the specific band of spikes correspond to one specific gyro-harmonic number and frequencies $f$ of these spikes are proportional to the magnetic field in their sources $(B \sim f)$. We note that all bands of spikes in BM model are generated in one region. Now, let us assume that the magnetic field in this region changes as $B=B_{0} \exp ^{-z / H}$, where $B_{0}$ is the magnetic field at some reference height in the solar atmosphere, $z$ is the height above this reference level and $H$ is the height scale of the magnetic field. Then we can re-sample the radio spectra expressed in frequencies to those expressed in heights $z(f)$ according to the formula $z(f)=-H \ln \frac{B}{B_{0}}=-H \ln \frac{f}{f_{0}}$, where $z(f)$ is the height in the solar atmosphere in dependence on frequency $f$. For the purpose of the present study, we selected the frequency band of $1370-1700 \mathrm{MHz}$ that corresponds to the blue band at 12:26:15 UT in Figure 7 and also covers the following narrow band of spikes (Figure 2a). In order to increase the signal/noise ratio we used the radio spectrum of this band with time resolution $0.2 \mathrm{~s}$. Taking $f_{0}$ $=1700 \mathrm{MHz}$, we re-sampled this radio spectrum from $\mathrm{f}(\mathrm{MHz})$ to $z$. Then the frequency profiles from these re- sampled radio spectrum were transform to the Fourier spectra and these Fourier spectra were averaged over time intervals of 2 seconds. This interval of $2 \mathrm{~s}$ is taken as a compromise between highly variable Fourier spectra at shorter intervals and only few spectra for longer time intervals that do not show the Fourier spectrum evolution with sufficiently good time resolution. As the result of these computations we obtained the power-law spectra with the power-law index that evolved in time as shown in Figure 8. Examples of these spectra at three selected times are shown in Figure 9. Here, the interval of spatial scales in the natural logarithm $\mathrm{LN}\left(\mathrm{k}_{z}\right)=$ $4.2-6.5$, where the power-law index was determined by the fitting techniques (black lines), is shown. As seen in Figure 8 the power-law index evolves in time. Firstly, at the time of strong broad band of spikes at 12:26:15 UT the Fourier spectrum (Figure 9) becomes very flat with the power-law index -0.8. Then the power-law index decreases up to -2.4 at 12:26:32 UT. After this decrease, there is the time interval of about 12:26:34 - 12:26:45 UT with broad band of spikes and the power-law index varying around the value $-1.66(-5 / 3)$. We note that averaging of the radio spectra over this interval gives the power-law index close to $-5 / 3$. In the following times the Fourier spectrum becomes steeper with the powerlaw index up to -2.75 . The final increase of the powerlaw index is given by signal decrease in comparison to instrumental noise. The present values of the powerlaw index are within the interval of the power-law indices found in the paper by Karlický et al. (2000) (-0.8 $--2.85)$. It also indicates that the uniqueness of this spike event is only in observations of these very narrow bands of spikes. We note that in our previous study we analyzed the whole $800-2000 \mathrm{MHz}$ range, neglecting the bands of spikes and considering different emission mechanism. On the contrary, here we have bands of spikes that are interpreted as generated on Bernstein modes. Therefore, we analyzed by this Fourier method only one band of spikes (1370-1700 MHz). Nevertheless, we interpret the power-law spectra by the same way as in our previous studies, i.e. the spikes are generated in the magnetohydrodynamic turbulence, that in the stationary state is described by the Kolmogorov spectral index $-5 / 3$. We think that the deviations of the found power-law indices from the Kolmogorov spectral index is given by the evolution of this turbulence and thus by deviations of this turbulence from the stationary state.

Now, considering these results, we summarize the processes generating the narrowband spikes in the 7 November 2013 event, as follows. In the flare impulsive phase, during the turbulent magnetic reconnection, electrons are accelerated as described in Dahlin et al. (2014); 
Guo et al. (2015); Zhou et al. (2016); Guo et al. (2020). Turbulent reconnection and turbulent reconnection outflows consist many magnetic traps of different sizes limited by magnetic mirrors. In these magnetic traps the accelerated electrons form the loss-cone distribution superimposed on the Maxwellian background plasma. As was shown by our computations these electrons generate the Bernstein modes with the gyro-harmonic numbers $s=3,4,5$ and 6 . Then these electrostatic Bernstein modes (BM) are transformed by their coalescence with the low-frequency waves or by the coalescence of the counterpropagating Bernstein modes into the electromagnetic waves as spikes on BM or double BM frequencies.

In turbulence that is in plasma reconnection outflows and/or in the flare turbulent magnetic reconnection, the plasma parameters vary not only due to the turbulence, but also due to gravitational stratification. Thus, in the regions with this turbulence, where magnetic traps are formed, the ratio of the electron plasma and electroncyclotron frequencies $Y=\omega_{\mathrm{pe}} / \omega_{\mathrm{ce}}$ varies in space and time. We think that individual spikes are generated inside the magnetic traps. When a region with the magnetic traps is small, i.e. with the very narrow interval of values of $Y$, then only narrow bands of spikes are generated as in the ending part of the 7 November 2013 event. On the other hand, for large regions with such traps, i.e. with the broad interval of values of $Y$, broad bands of spikes can be observed, see e.g. the broad bands of spikes in Figure 1 in the 12:26:09 - 12:26:39 UT interval. In turbulence the magnetic traps have different sizes and thus individual spikes have different frequency bandwidths. We think that this relation between sizes of the magnetic traps (in correspondence with spatial scales in turbulence) and the frequency bandwidths of spikes explains why the Fourier analysis of the frequency profiles of the spike events show the power-law spectra.

\section{CONCLUSIONS}

We analyzed the narrowband spikes observed in the 800-2000 MHz range during the impulsive phase of the November 7, 2013 flare. These spikes started as typical spikes clustered in broad bands and then the frequency distribution of this spikes in the radio spectrum changed to four very narrow bands of spikes. We focused our attention to these very narrow bands. We successfully fitted frequencies of these bands by those calculating dispersion branches and growth rates of the Bernstein modes. Using this model and taking the spike band frequencies as BM frequencies we estimated the plasma density and magnetic field strength in the narrowband spike source as $n_{\mathrm{e}}=9.02 \times 10^{9} \mathrm{~cm}^{-3}$ and $B=113 \mathrm{G}$, respectively. On the other hand, for the radio emission on the double BM frequencies the values of the plasma density and magnetic field strength are 4 and 2 times lower, respectively. We also considered the UHW model (Stepanov et al. 1999; Bárta \& Karlický 2001). Comparing the both models we presented arguments in favor of the BM model. Namely, we succeeded (almost perfectly) to fit BM frequencies with the observed band structure as expected in the BM model. Moreover, the frequency difference among three bands was close to 300 $\mathrm{MHz}$. It also speaks in favor of the BM model, because it is not very probable that UHW model with three sources at different locations give such a result. Based on these arguments, we concluded that the BM model, proposed by Willes \& Robinson (1996), explains this spike event.

The presented spike event shows a transition from typical spike event with the broadband clouds of spikes to very narrow spike bands. It shows that the BM model, that successfully fitted the narrow bands of spikes in the November 7, 2013 event, can be also valid at least for some group of spike events. However, we think that UHW model or direct ECM models cannot be excluded in some other spike events.

In the Fourier analysis of the frequency profiles of the November 7, 2013 event we found the power-law spectra with the power-law indices in the $-0.80--2.75$ interval. Because at some time interval this power-law index was close to the Kolmogorov index $(-5 / 3)$, we interpreted these power-law spectra in a scenario where the narrowband spikes are generated in turbulent plasma reconnection outflows or/and directly in the turbulent magnetic reconnection of solar flares.

\section{ACKNOWLEDGMENTS}

M.K. and J.B acknowledge support from the project RVO-67985815 and GA ČR grants 19-09489S, 2009922J, 20-07908S and the financial support by the German Science Foundation (DFG) via the projects BU777-17-1. This work was supported by The Ministry of Education, Youth and Sports from the Large Infrastructures for Research, Experimental Development and Innovations project "e-Infrastructure CZ LM2018140". J.R. acknowledges support by the Science Grant Agency project VEGA 2/0048/20 (Slovakia). Help of the Bilateral Mobility Project SAV-18-01 of the SAS and CAS is acknowledged as well. We also thank the anonymous referee whose comments helped to improve this paper.

\section{APPENDIX A}

The dispersion branches of the electrostatic waves are computed using the plasma permittivity tensor $\epsilon_{\|}$. We assume that plasma consists of cold background 
Maxwellian electrons of density $n_{\mathrm{e}}$ and loss-cone hot electrons with density $n_{\mathrm{h}}$, where $n_{\mathrm{e}} \gg n_{\mathrm{h}}$. Hot electrons have (DGH) distribution function for $j=1$ (Dory et al. 1965)

$$
f_{\text {hot }}\left(u_{\|}, u_{\perp}\right)=\frac{u_{\perp}^{2}}{2(2 \pi)^{3 / 2} v_{\mathrm{t}}^{5}} \exp \left(-\frac{u_{\perp}^{2}+u_{\|}^{2}}{2 v_{\mathrm{t}}^{2}}\right),
$$

where $u_{\|}, u_{\perp}$ are the velocities parallel and perpendicular to the magnetic field, respectively. $v_{\mathrm{t}}$ is the characteristic velocity. All ions have same temperature as cold electrons. In our case, ions do not significantly contribute to the permittivity for frequencies $\omega \sim \omega_{\text {pe }}$, where $\omega_{\text {pe }}$ is the electron plasma frequency.

For condition $n_{\mathrm{h}} \ll n_{\mathrm{e}}$, the permittivity can be separated into the permittivity $\epsilon_{\|}^{(0)}$ for cold electrons and the permittivity $\epsilon_{\|}^{(1)}$ connected with hot electrons (Chen 1974; Zheleznyakov 1997; Fitzpatrick 2015). In this approximation, solutions for the electrostatic waves are given only by the term $\epsilon_{\|}^{(0)}=0$. The relation for dispersion branches of the Bernstein modes can be written in the form

$$
\begin{gathered}
\epsilon_{\|}^{(0)}=1-2 \omega_{\mathrm{pe}}^{2} \frac{e^{-\lambda}}{\lambda} \sum_{l=1}^{\infty} \frac{l^{2} I_{l}(\lambda)}{\omega^{2}-l^{2} \omega_{\mathrm{ce}}^{2}}=0, \\
\omega_{\mathrm{pe}}^{2}=\frac{n_{\mathrm{e}} e^{2}}{m_{\mathrm{e}} \epsilon_{0}}, \quad \lambda=\frac{k_{\perp}^{2} v_{\mathrm{tb}}^{2}}{\omega_{\mathrm{ce}}^{2}},
\end{gathered}
$$

where $\epsilon_{0}$ is the permittivity of free space, $\omega_{\text {ce }}$ is the the electron cyclotron frequency, $\mathbf{k}=\left(k_{\|}, k_{\perp}\right)$ is the wave vector parallel and perpendicular to the direction of the magnetic field, respectively, $\omega$ is the wave frequency of the electrostatic wave, $I_{l}(\lambda)$ is the modified Bessel function of $l$ th order, $\lambda$ is the dimensionless parameter, $m_{\mathrm{e}}$ is the electron mass, $e$ is the electron charge.

We searched roots (dispersion branches) directly from Equation 2 using Python and Scipy library ${ }^{2}$; we do not use any approximative analytical solutions. Specifically, we search for roots using the Levenberg-Marquardt damped root method (Levenberg 1944; Marquardt 1963; Moré et al. 1980; Press et al. 2007) and following the method by Benáček \& Karlický (2019). Our method divides the examined $\omega-k_{\perp}$ domain into a rectangular grid. Each grid cell then serves as a starting point in the root search algorithm. We used $10^{3}$ starting points in $\omega$ and 400 starting points in $k_{\perp}$ direction. Because the branches can be usually assumed as horizontal, the minimalization is made in variable $\omega$. The minimalization procedure has the eventual error $<10^{-8} \omega_{\mathrm{pe}}$. We

2 python.org, scipy.org found that for such an error, sum over harmonic number $l \leq 30$ is sufficient. Consequently, all the found roots from all starting points are aggregated, giving the resulting dispersion branches.

Generally, the electrostatic (Bernstein and upperhybrid) waves can be unstable if the double plasma resonance condition is fulfilled

$$
\omega-\frac{k_{\|} u_{\|}}{\gamma_{\mathrm{rel}}}-\frac{s \omega_{\mathrm{ce}}}{\gamma_{\mathrm{rel}}}=0
$$

where $\gamma_{\mathrm{rel}}=\left(1-v^{2} / c^{2}\right)^{-\frac{1}{2}}$ is the relativistic Lorentz factor and $s$ is the resonance gyro-harmonic number. Whether amplitude of an electrostatic wave increases for some $\left(\omega, k_{\perp}\right)$ we need to calculate the growth rate (Zheleznyakov \& Zlotnik 1975a)

$$
\begin{gathered}
\gamma\left(\omega, k_{\perp}\right)=-\frac{\operatorname{Im} \epsilon_{\|}^{(1)}}{\left[\frac{\partial \operatorname{Re} \epsilon_{\|}^{(0)}}{\partial \omega}\right]_{\epsilon_{\|}^{(0)}=0}}, \\
\frac{\partial \epsilon_{\|}}{\partial \omega}=4 \omega \omega_{\mathrm{pe}}^{2} \frac{e^{-\lambda}}{\lambda} \sum_{l=1}^{\infty} \frac{l^{2} I_{l}(\lambda)}{\left(\omega^{2}-l^{2} \omega_{\mathrm{ce}}^{2}\right)^{2}} .
\end{gathered}
$$

The explicit expression for this growth rate was provided by Kuznetsov (2005). The imaginary part of the permittivity can be written as follows

$$
\begin{gathered}
\operatorname{Im}\left(\epsilon_{\|}^{(1)}\right)=-2 \pi^{2} m_{\mathrm{e}}^{4} \frac{\omega_{\mathrm{pe}}^{2}}{k^{2}} \sum_{l=s+1}^{\infty} a b^{2} \\
\times \int_{0}^{\pi} J_{l}\left(\frac{\gamma_{\mathrm{rel}} k_{\perp} v_{\perp}}{\omega_{\mathrm{ce}}}\right) \frac{\gamma_{\mathrm{rel}}^{5} \sin \phi}{\frac{\partial \psi}{\partial \rho}} \frac{l \omega_{\mathrm{ce}}}{\gamma_{\mathrm{rel}} v_{\perp}} \frac{\partial f}{\partial p_{\perp}} \mathrm{d} \phi \\
\frac{\partial \psi}{\partial \rho}=\frac{\gamma_{\mathrm{rel}}^{2} l \omega_{\mathrm{ce}}}{c^{2}}\left(v_{\|}^{2}+v_{\perp}^{2}\right) \\
v_{\|}=-a \cos (\phi), \quad v_{\perp}=b \sin (\phi) \\
a^{2}=\frac{l^{2} \omega_{\mathrm{ce}}^{2} c^{2}\left(l^{2} \omega_{\mathrm{ce}}^{2}-\omega^{2}\right)}{l^{4} \omega_{\mathrm{ce}}^{4}}
\end{gathered}
$$

As positions of the growth rates in Figure 5 are given only by hot electrons, we can compute the growth rates independently on position of dispersion branches in the whole domain $\omega-k_{\perp}$ using Equation 7 . We use 600 grid points in $\omega$ direction, and 300 points in $k_{\perp}$ direction. The growth rates are overlaid by solutions of the dispersion equation (Equation 2) that represent the Bernstein modes used in the interpretation of spikes in the model by Willes \& Robinson (1996).

On the other hand, in the interpretation of spikes generated at the upper-hybrid frequency, we compute the growth rates in Figure 6 as follows: First, for a given 
ratio $\omega_{\mathrm{UH}} / \omega_{\text {ce }}$ a branch of the upper-hybrid waves is calculated from the relation $\omega_{\mathrm{UH}}=\sqrt{\omega_{\mathrm{pe}}^{2}+\omega_{\mathrm{ce}}^{2}+3 k_{\perp}^{2} v_{\mathrm{tb}}^{2}}$. Then for this branch the growth rates from Equation 7 are computed and among them the maximal growth rate

$$
\Gamma\left(\frac{\omega_{\mathrm{UH}}}{\omega_{\mathrm{ce}}}\right)=\max \left\{\gamma_{\mathrm{UH}}\left(\frac{\omega_{\mathrm{UH}}}{\omega_{\mathrm{ce}}}, \omega, k_{\perp}\right)\right\},
$$

is taken as a value for the given ratio $\omega_{\mathrm{UH}} / \omega_{\mathrm{ce}}$.

\section{REFERENCES}

Aschwanden, M. J. 1990, A\&AS, 85, 1141

Aschwanden, M. J. 2002, SSRv, 101, 1

Aschwanden, M. J. 2004, Physics of the Solar Corona. An Introduction, Springer, Praxis Publ., UK

Aschwanden, M. J., Dennis, B. R., \& Benz, A. O. 1998, ApJ, 497, 972

Bárta, M., Büchner, J., Karlický, M., \& Kotrč, P. 2011a, ApJ, 730, 47

Bárta, M., Büchner, J., Karlický, M., \& Skála, J. 2011b, ApJ, 737, 24

Bárta, M., \& Karlický, M. 2001, A\&A, 379, 1045

Benáček, J., \& Karlický, M. 2019, ApJ, 881, 21

Benz, A. O. 1986, SoPh, 104, 99

Benz, A. O., Messmer, P., \& Monstein, C. 2001, A\&A, 366, 326

Benz, A. O., Monstein, C., Beverland, M., Meyer, H., \& Stuber, B. 2009, SoPh, 260, 375

Benz, A. O., Saint-Hilaire, P., \& Vilmer, N. 2002, A\&A, 383, 678

Bhattacharjee, A., Huang, Y.-M., Yang, H., \& Rogers, B. 2009, Physics of Plasmas, 16, 112102

Bouratzis, C., Hillaris, A., Alissandrakis, C. E., et al. 2016, A\&A, 586, A29

Chen, F. F. 1974, Introduction to plasma physics, Plenum Press, New York

Dabrowski, B. P., \& Benz, A. O. 2009, A\&A, 504, 565

Dahlin, J. T., Drake, J. F., \& Swisdak, M. 2014, Physics of Plasmas, 21, 092304

Dory, R. A., Guest, G. E., \& Harris, E. G. 1965, Physical Review Letters, 14, 131

Drake, J. F., Arnold, H., Swisdak, M., \& Dahlin, J. T. 2019, Physics of Plasmas, 26, 012901

Droege, F. 1977, A\&A, 57, 285

Feng, S. W. 2019, Ap\&SS, 364, 4

Fitzpatrick, R. 2015, Plasma physics: an introduction (CRC Press)

Fleishman, G. D. 2004, ApJ, 601, 559

Fleishman, G. D., Gary, D. E., \& Nita, G. M. 2003, ApJ, 593,571

Fleishman, G. D., \& Mel'nikov, V. F. 1998, Physics Uspekhi, 41, 1157
Fu, Q., Li, C., \& Jin, S. 1985, in Solar Physics and Interplanetary Travelling Phenomena, ed. C. de Jager \& B. Chen, Vol. 1, 560

Guedel, M. 1990, A\&A, 239, L1

Guo, F., Liu, Y.-H., Daughton, W., \& Li, H. 2015, ApJ, 806,167

Guo, F., Liu, Y.-H., Li, X., et al. 2020, Physics of Plasmas, 27, 080501

Isliker, H., \& Benz, A. O. 1994, A\&AS, 104, 145

Jiřička, K., \& Karlický, M. 2008, SoPh, 253, 95

Jiřička, K., Karlický, M., Kepka, O., \& Tlamicha, A. 1993, SoPh, 147, 203

Jiřička, K., Karlický, M., Mészárosová, H., \& Snížek, V. 2001, A\&A, 375, 243

Karlický, M. 1984, SoPh, 92, 329

Karlický, M., \& Bárta, M. 2011, ApJ, 733, 107

Karlický, M., Jiřička, K., \& Sobotka, M. 2000, SoPh, 195, 165

Karlický, M., \& Rybák, J. 2020, ApJS, 250, 31

Karlický, M., Sobotka, M., \& Jiřička, K. 1996, SoPh, 168, 375

Karlický, M., Rybák, J., \& Monstein, C. 2018, SoPh, 293, 143

Karlický, M., \& Yasnov, L. V. 2015, A\&A, 581, A115

Karlický, M., \& Yasnov, L. V. 2018, ApJ, 867, 28

Karlický, M., \& Yasnov, L. 2020, A\&A, 638, A22

Krucker, S., \& Benz, A. O. 1994, A\&A, 285, 1038

Krueger, A. 1979, Introduction to solar radio astronomy and radio physics, D. Reidel Publ. Comp., Dordrecht, Holland

Kuijpers, J., van der Post, P., \& Slottje, C. 1981, A\&A, 103, 331

Kuznetsov, A. A. 2005, A\&A, 438, 341

Lazarian, A., Eyink, G. L., Jafari, A., et al. 2020, Physics of Plasmas, 27, 012305

Lazarian, A., \& Vishniac, E. T. 1999, ApJ, 517, 700

Lemen, J. R., Title, A. M., Akin, D. J., et al. 2012, SoPh, 275,17

Levenberg, K. A 1944, Quart. Appl. Math. 2, 164-168

Loureiro, N. F., Schekochihin, A. A., \& Cowley, S. C. 2007, Physics of Plasmas, 14, 100703

Marquardt, D. 1963, SIAM J. Appl. Math. 11, 431-441 
McLean, D. J., \& Labrum, N. R. 1985, Solar radiophysics : studies of emission from the sun at metre wavelengths, Cambridge University Press, Cambridge, London

Melrose, D. B. 2017, Reviews of Modern Plasma Physics, 1, 5

Melrose, D. B., \& Dulk, G. A. 1982, ApJ, 259, 844

Messmer, P., \& Benz, A. O. 2000, A\&A, 354, 287

Moré, J. J. Garbow, B. S. \& Hillstrom, K. E. 1980, User guide for MINPACK-1, Argonne Nat. Lab., ANL-80-74, http://cds.cern.ch/record/126569

Mészárosová, H., Veronig, A., Zlobec, P., \& Karlický, M. 2003, A\&A, 407, 1115

Ni, S., Chen, Y., Li, C., et al. 2020, ApJL, 891, L25

Nita, G. M., Fleishman, G. D., Gary, D. E., Marin, W., \& Boone, K. 2014, ApJ, 789, 152

Pesnell, W. D., Thompson, B. J., \& Chamberlin, P. C. 2012, SoPh, 275, 3

Press, W. H. Teukolsky, S. A. Vetterling, W. T. \& Flannery, B. P. 2007, Numerical Recipes 3rd Edition: The Art of Scientific Computing, Cambridge University Press, New York, NY, USA

Priest, E., \& Forbes, T. 2000, Magnetic Reconnection, Cambridge, UK: Cambridge University Press

Priest, E. 2014, Magnetohydrodynamics of the Sun, by Eric Priest, Cambridge, UK: Cambridge University Press, 2014. doi:10.1017/CBO9781139020732
Rozhansky, I. V., Fleishman, G. D., \& Huang, G. L. 2008, ApJ, 681, 1688

Staehli, M., \& Magun, A. 1986, SoPh, 104, 117

Stepanov, A. V., Kliem, B., Krüger, A., Hildebrand t, J., \& Garaimov, V. I. 1999, ApJ, 524, 961

Tajima, T., Benz, A. O., Thaker, M., \& Leboeuf, J. N. 1990, ApJ, 353, 666

Vlahos, L., \& Sharma, R. R. 1985, ApJ, 290, 347

Wentzel, D. G. 1991, ApJ, 373, 285

Willes, A. J., \& Robinson, P. A. 1996, ApJ, 467, 465

Winglee, R. M., Dulk, G. A., \& Pritchett, P. L. 1988, ApJ, 328,809

Yasnov, L. V., Benáček, J., \& Karlický, M. 2019, SoPh, 294, 29. doi:10.1007/s11207-019-1415-9

Zhou, X., Büchner, J., Bárta, M., Gan, W., \& Liu, S. 2016, ApJ, 827, 94

Zheleznyakov, V. V. 1997, Radiation in astrophysical plasmas [in Russian]; Original Russian Title "Izlucheniye v astrofizicheskoy plasme"

Zheleznyakov, V. V. \& Zlotnik, E. I. 1975a, Sol. Phys., 43, 431

Zlobec, P., \& Karlický, M. 1998, SoPh, 182, 477

Zlotnik, E. Y. 2013, SoPh, 284, 579 

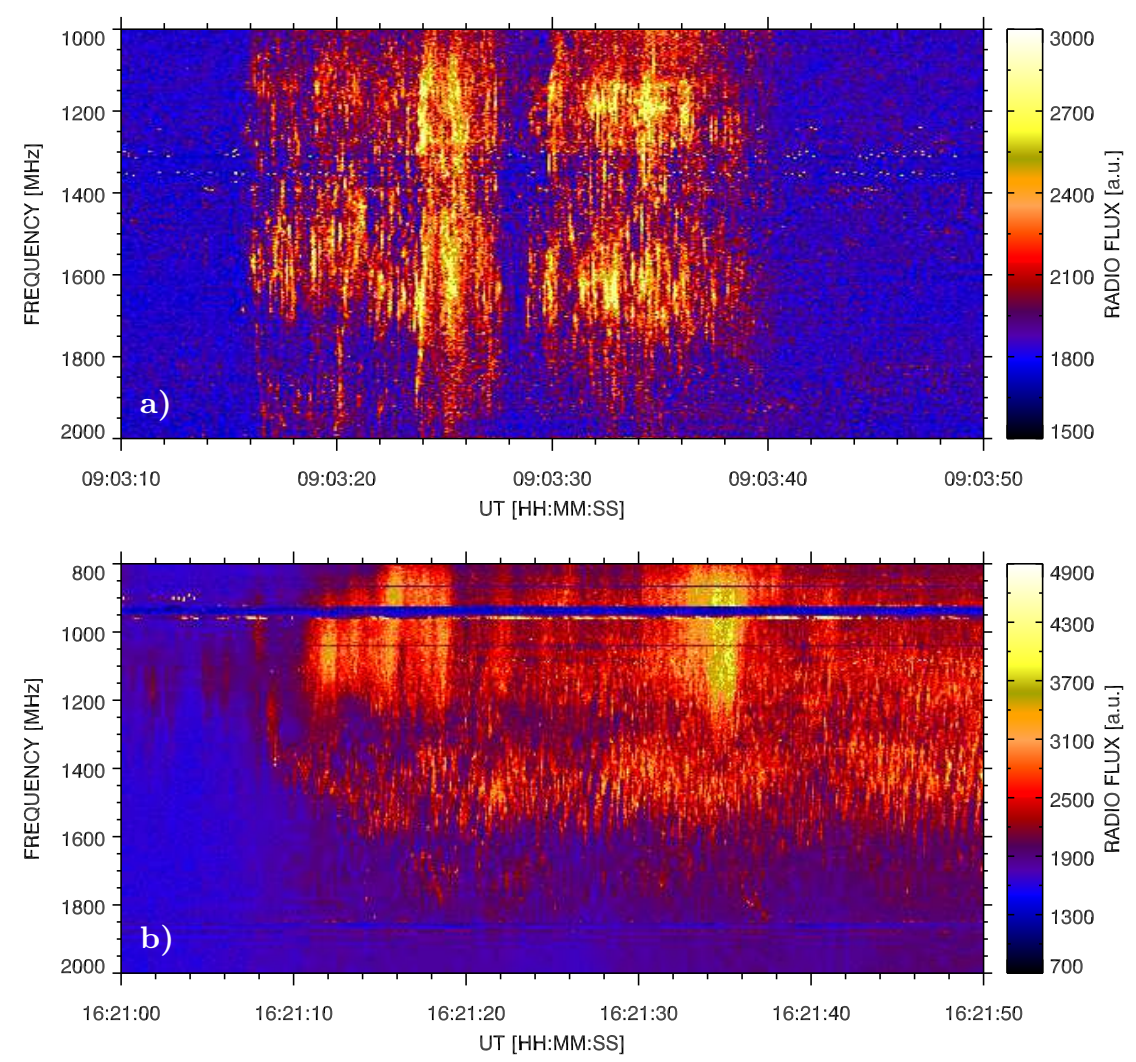

Figure 1. Examples of the narrowband spikes observed in the 14 September 1992 (a) and 10 June 2003 (b) flares. The horizontal narrow band in the $940-970 \mathrm{MHz}$ range of the 10 June 2003 spectrum means a gap in observations. 

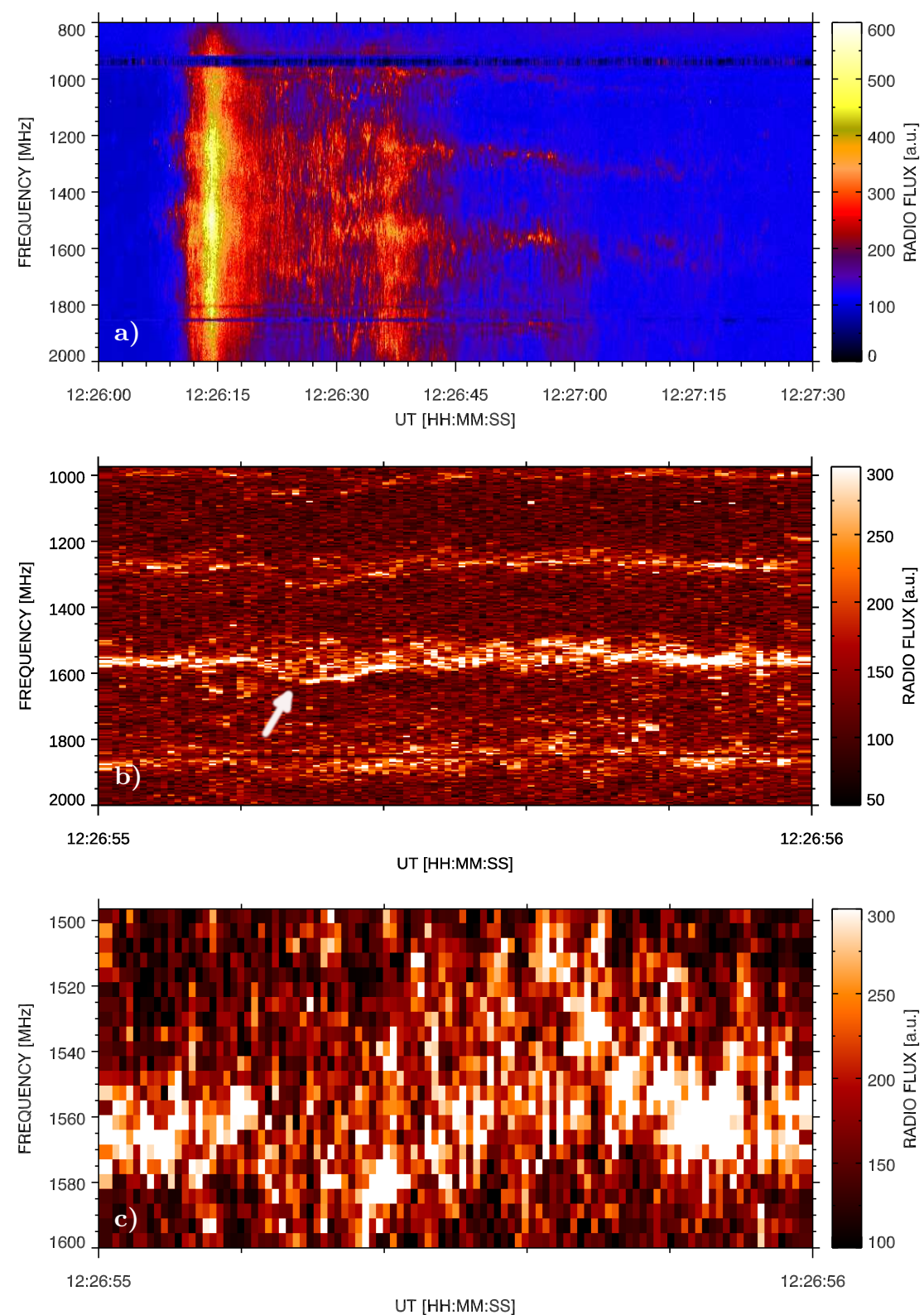

Figure 2. a) Radio spectrum showing the narrowband spikes that started as typical spike event with spikes in broad bands (12:26:09 - 12:26:39 UT) and followed by unique, four very narrow bands of spikes observed on 7 November 2013. In the 940-970 $\mathrm{MHz}$ range there is a gap in observations. b) Detail of this radio spectrum in the 1000-2000 MHz range starting at 12:26:55 UT, lasting $1 \mathrm{~s}$ and showing the narrow bands of spikes. The time resolution is $0.01 \mathrm{~s}$. The arrow shows the band frequency variation which the form is synchronized with the similar forms in all four bands. The spectrum is not smoothed and thus showing bins in the record. c) The same as b), but in the 1500-1600 MHz range, showing bright bins (roughly spikes) within one narrow band of spikes. 

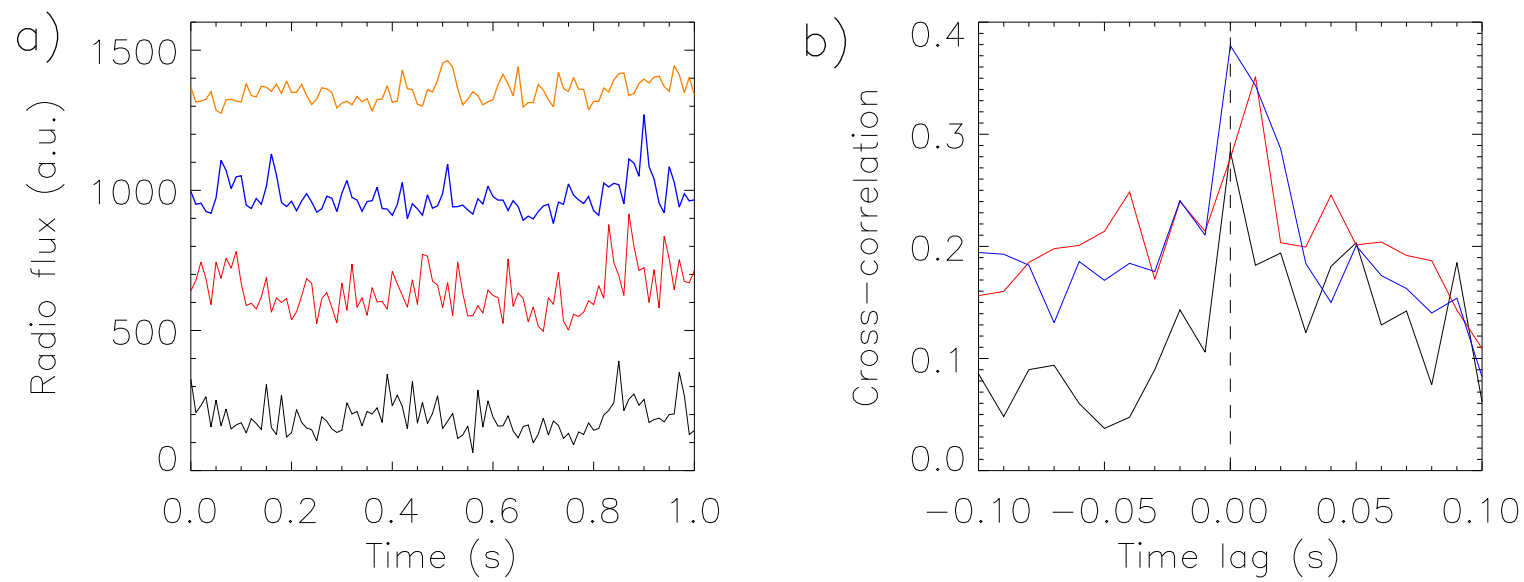

Figure 3. a) Radio flux in dependence on time at $1877 \mathrm{MHz}$ (black line), at $1572 \mathrm{MHz}$ (red line) + 400 a.u., at $1276 \mathrm{MHz}$ (blue line) + 800 a.u., and at $1003 \mathrm{MHz}$ (orange line) + 1200 a.u.. starting at 12:26:55 UT and lasting 1 s. b) Cross-correlations of the radio flux profiles on 1877 and $1572 \mathrm{MHz}$ (black line), 1572 and $1276 \mathrm{MHz}$ (red line), and 1276 and $1003 \mathrm{MHz}$ (blue line).
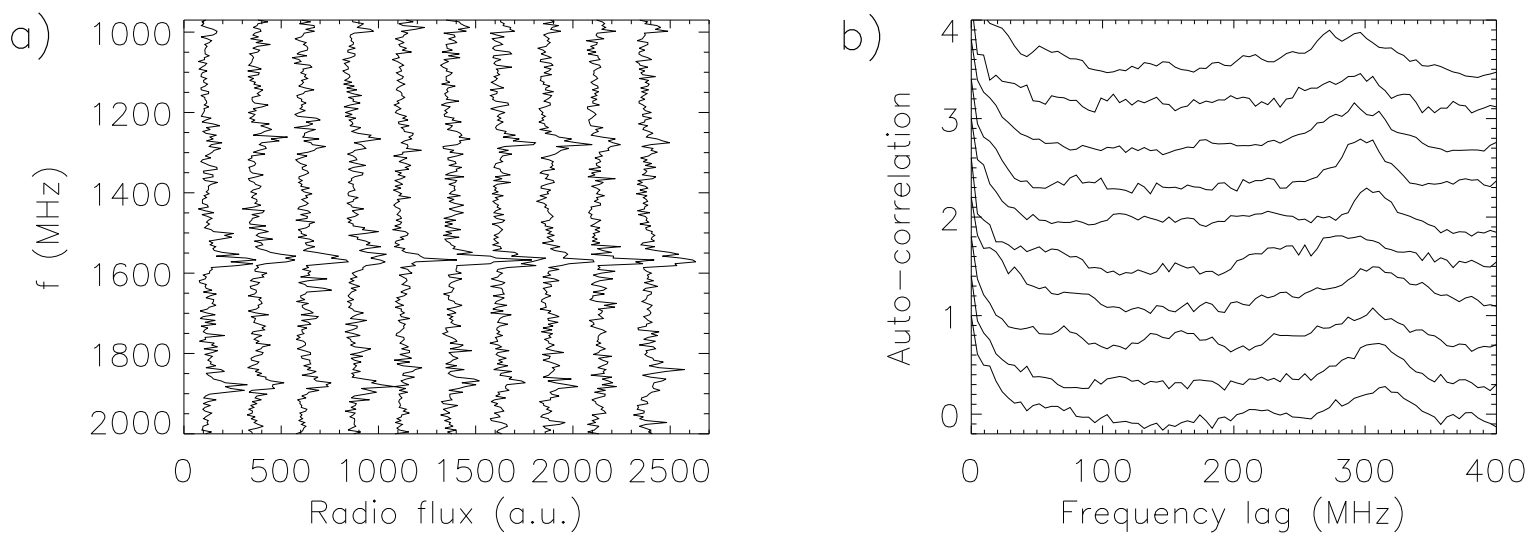

Figure 4. a) Radio flux in dependence on frequency in 10 instants starting at 12:26:55 UT, each after 0.01 s, corresponding to the radio flux shift 250 a.u. b) Auto-correlations of the radio flux profiles shown in (a). The shift of each auto-correlation is 0.4 . 


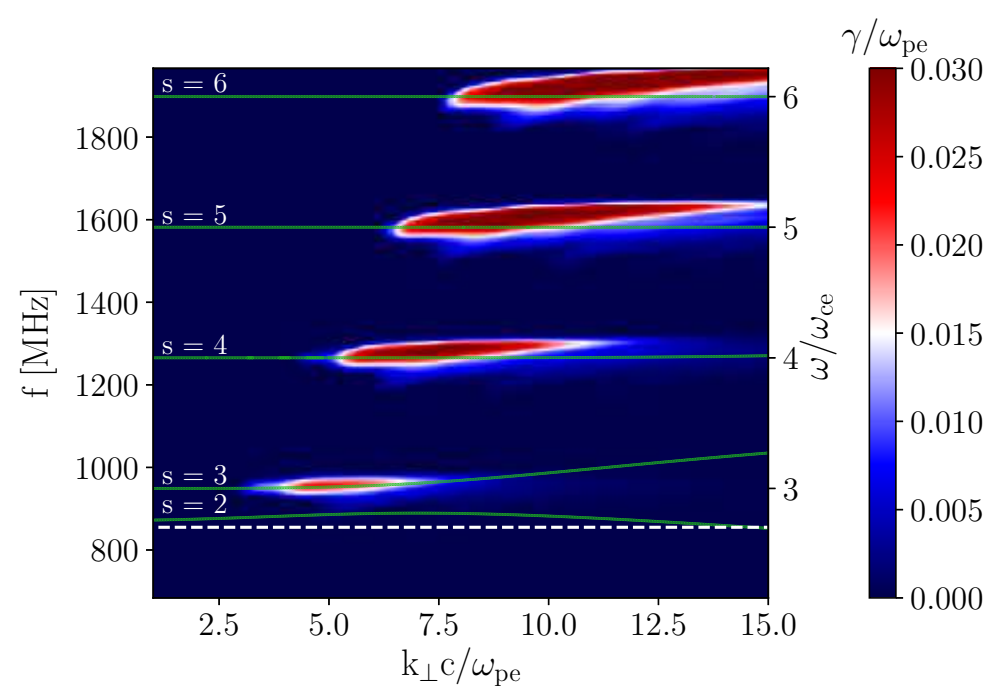

Figure 5. Growth rates of the Bernstein modes as a function of the frequency and perpendicular k-wave vector for parameters $\omega_{\mathrm{pe}} / \omega_{\mathrm{ce}}=2.7, v_{\mathrm{tb}} / c=0.02, v_{\mathrm{t}} / c=0.25, n_{\mathrm{e}} / n_{\mathrm{h}}=10$. The values of $\mathrm{f}$ in $\mathrm{MHz}$ correspond to the radio emission on BM frequencies, i.e. $\omega_{\mathrm{pe}}=2 \pi f_{\mathrm{pe}}, f_{\mathrm{pe}}=855 \mathrm{MHz}$. For the radio emission on double BM frequencies the values of $\mathrm{f}$ need be divided by 2 and $f_{\mathrm{pe}}$ $=427.5 \mathrm{MHz}$. Green lines: Dispersion branches computed from the Equation 2, $s$ is the gyro-harmonic number of each branch. White dashed horizontal line: The plasma frequency.

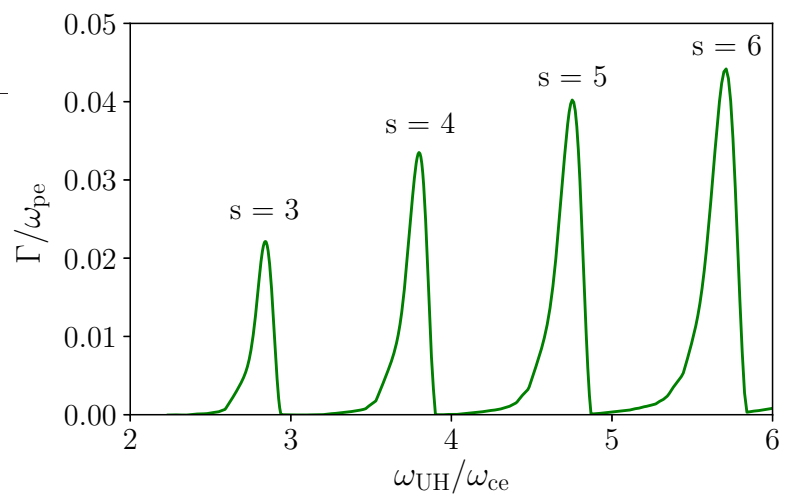

Figure 6. Growth rate of the upper-hybrid waves in dependence on the $\omega_{\mathrm{UH}} / \omega_{c e}$ ratio, $s$ denotes the gyro-harmonic number. 


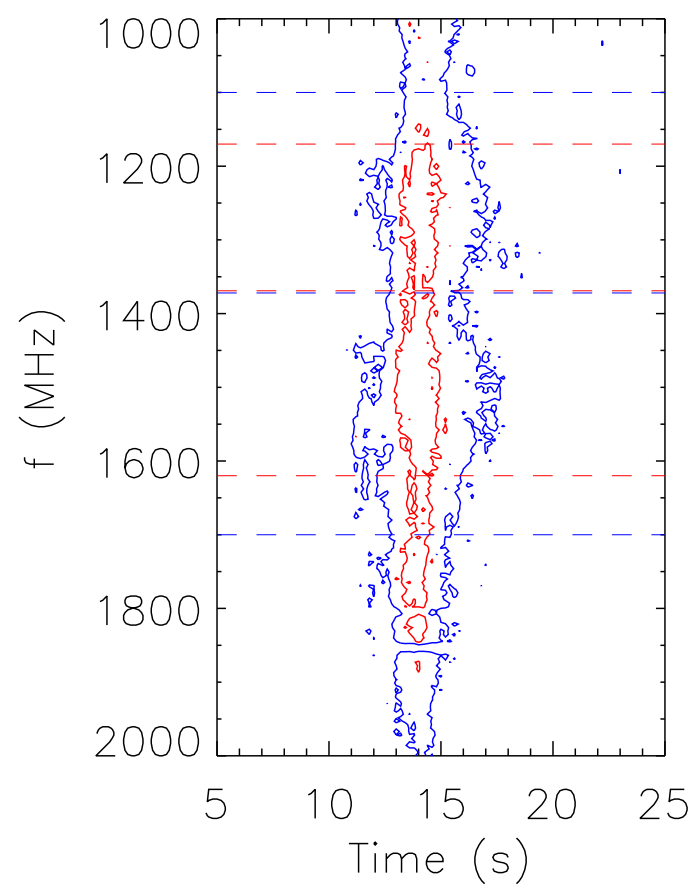

Figure 7. Blue and red contours of spikes at two levels (320 a.u. and 440 a.u.) at 12:26:05-12:26:25 UT (Time (s) after 12:26:00 UT), compare with spikes in Figure 2a. Blue and red horizontal dashed lines limit the frequency width of two bands of spikes as $1100-1370 \mathrm{MHz}$ and $1370-1700 \mathrm{MHz}$, and as $1170-1370 \mathrm{MHz}$ and $1370-1620 \mathrm{MHz}$, respectively.

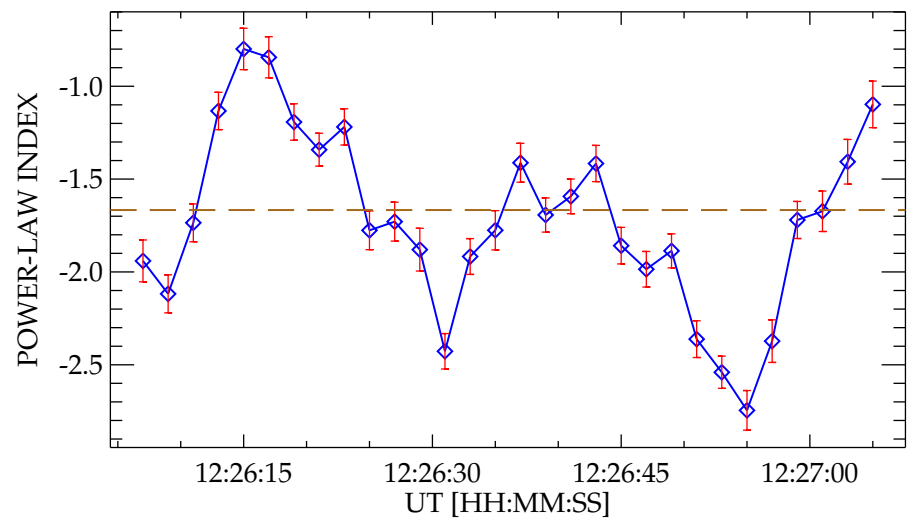

Figure 8. Time evolution of the power-law index of the Fourier spectra of the 1370-1700 MHz frequency profiles during the November 7, 2013 event. The horizontal dashed line corresponds to the power-law index $-5 / 3$. 


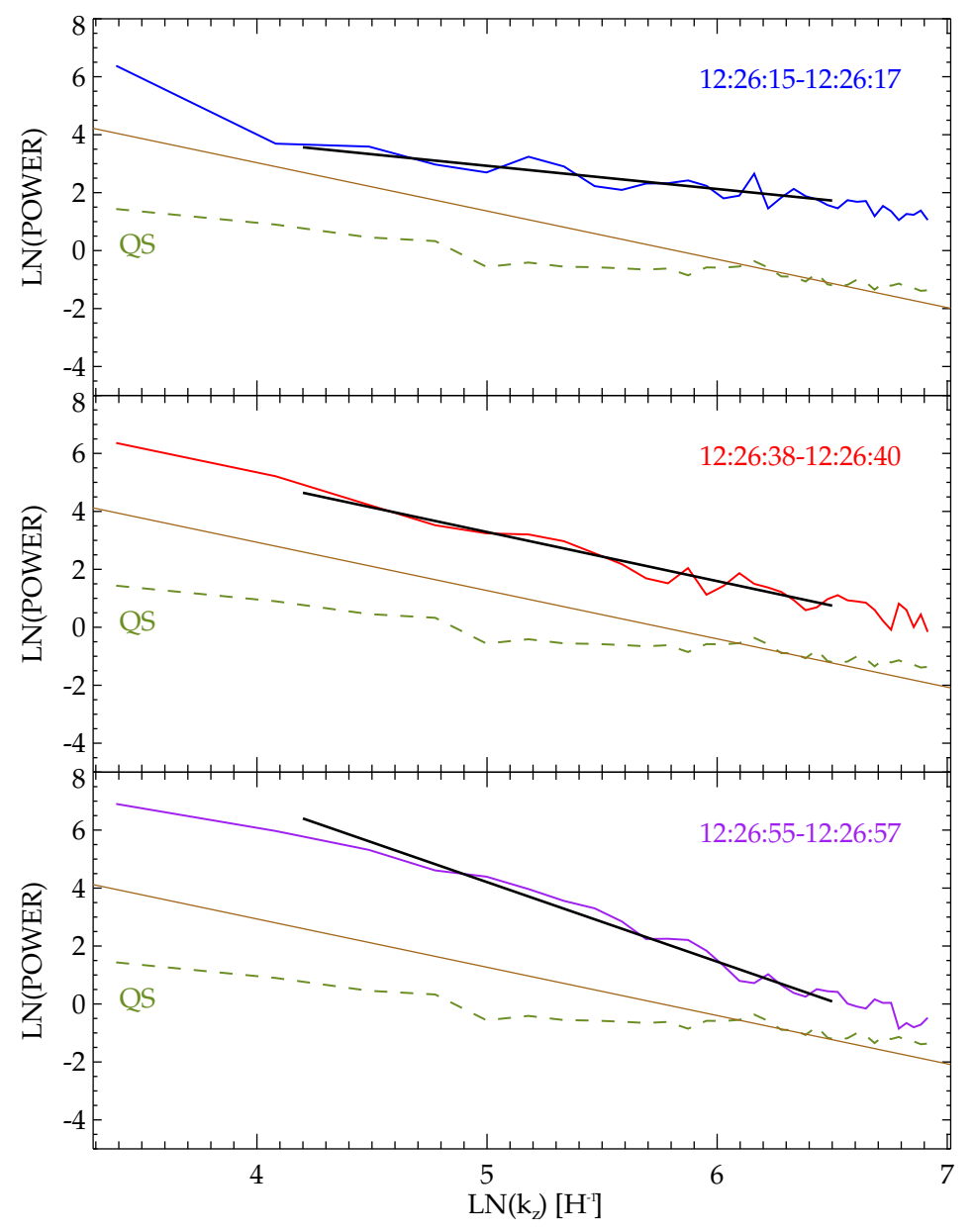

Figure 9. Fourier spectra of the 1370-1700 MHz frequency profiles integrated over $2 \mathrm{~s}$ at three instants 12:26:15 UT (blue line), 12:26:38 UT (red line) and 12:26:55 UT (violet line), in the natural logarithmic scales (LN) together with the spectrum of the frequency profile without the emission before the spike event (QS - quiet Sun) at 12:26:00 UT (olive dashed line). Black straight lines mean the fitted lines of the Fourier spectrum in the interval of $\mathrm{LN}\left(\mathrm{k}_{z}\right)=4.2-6.5$. The olive straight lines denote the lines with the power-law index $-5 / 3$. 